\title{
Obstacles to the Development of BIM in China's Prefabricated Construction: A Decision-making Test and Evaluation Laboratory (DEMATEL) Model
}

\author{
Yanan Ma, Qiong Shen*, and Lei Tian \\ College of Architecture and Urban-Rural Planning, Sichuan Agricultural University, \\ Dujiangyan Sichuan, 611830, China
}

\begin{abstract}
With the continuous progress of the time, technology is also developing rapidly, among which Building Information Modeling (BIM) has also become a hot topic in prefabricated constructions. Previous research has made it clear that BIM can promote the development of prefabricated construction. However, in practical applications, BIM technology has not been well developed in prefabricated construction. In order to find out the obstacles to the development of BIM technology in China's prefabricated construction, and identify the relationship between obstacles, this study identified 20 obstacles through literature review. This study used the Decision-making Test and Evaluation Laboratory (DEMATEL) model to get the importance of each obstacle and the causality between them, and provides a solution to the development of BIM technology in China to promote the development of BIM technology in new construction methods. Keywords: BIM, Prefabricated construction, Obstacles.
\end{abstract}

\section{Introduction}

In the 1980s, with the proposal of building energy efficiency, green buildings are gradually emerging in China. The traditional cast-in-place building model consumes more energy than green building, and it is also likely to have a greater impact on the surrounding environment [1], which does not meet China's requirements for sustainable and green development. The appearance of prefabricated construction conforms to the requirements of green building, which embodies the advantages of green building. It has the advantages of energy-saving, environmental protection, material saving, reduction of labour and so on [2]. In order to face the urgent needs of all parts of the country to transform and upgrade to the modernization of the construction industry, the state and industry have successively issued relevant guidelines and policies. Since 2018, China's prefabricated construction market has grown rapidly. According to national policy directives, it is estimated that the national prefabricated construction market will have a space of about 2 trillion by 2020 . Newly assembled buildings accounted for $15 \%$ of new buildings, and further reached $30 \%$ in 2025 . With the improvement

\footnotetext{
* Corresponding author: swydong@sicau.edu.cn
} 
of technology and management level, prefabricated construction will have a broad market and space in China.

In the context of the continuous progress of the times, BIM technology has gradually become familiar with China's construction industry. It can help various professions in the construction industry to implement the integration of construction information. So it can improve the construction efficiency of prefabricated buildings, which is beneficial to development [3]. Because the development of BIM conforms to the principle that the more buildings are built off site and then onsite, the more labor and material costs are saved [4]. There are many advantages to using BIM technology in prefabricated construction. However, according to the perspective of development status, the situation of BIM in China is not optimistic. BIM is facing not only domestic application in prefabricated construction, but also obstacles to its implementation [5]. Scholars such as Oesterreich and Hong separately analyzed the development of BIM and the obstacles to the implementation of prefabricated construction $[6,7]$. Some scholars have linked the two together. For example, Tan analyzed the relationship between BIM's obstacles to the development of prefabricated construction [8]. However, there are not enough obstacles involved in these studies. Due to the influence of analytical methods, some factors that are considered not important would be screened out. Therefore, the purpose of this study is to expand the number of obstacles based on previous research, and use DEMATEL model to find the most important obstacles. Then analyzed the causal relationship between obstacles. According to the results, this study analyzed the obstacles to the development of BIM in China's prefabricated buildings and gave appropriate suggestions.

\section{Obstacles to BIM applications in prefabricated construction}

Based on the development status of BIM in prefabricated construction, it is necessary to analyze the obstacles to the development of BIM in prefabricated construction. In this study, the literature review method was used to check, compare, analyze and sort out the related obstacles. And on this basis, the existing unhealthy industrial phenomena are improved, and the obstacles summarized are made into expert questionnaires. Consultation and interviews were conducted with experts who have been engaged in related research and work for more than 3 years, and the obstacles were modified and supplemented. Finally, 20 obstacles were obtained, which are representative of the BIM implementation in prefabricated buildings, as showed in Table 1. 
Table 1. Obstacles are affecting development of BIM in prefabricated construction.

\begin{tabular}{clc}
\hline Number & \multicolumn{1}{c}{ Obstacles } & Related references \\
\hline F1 & High precision requirements for fabricated components & {$[8]$} \\
F2 & Lack of motivation and promotion & {$[8]$} \\
F3 & Lack of insurance applicable to BIM implementation & {$[8]$} \\
F4 & Lack of intellectual property protection & {$[8]$} \\
F5 & New construction methods take a long time to learn & {$[8]$} \\
F6 & High initial costs & {$[9]$} \\
F7 & Lack of uniform industry guidance standards & {$[9]$} \\
F8 & The construction industry is dispersed & {$[9]$} \\
F9 & Lack of standards to quantify BIM benefits & {$[9]$} \\
F10 & Conservative industry culture and resistance from client & {$[9]$} \\
F11 & Stakeholders' negative treatment & {$[10]$} \\
F12 & Lack of experienced practitioners & {$[10]$} \\
F13 & Lack of collaborative work processes & {$[10]$} \\
F14 & Lack of professional interaction & {$[10]$} \\
F15 & Lack of standard contract templates & {$[10]$} \\
F16 & Resistance to data sharing & {$[10]$} \\
F17 & Compatibility of BIM software & {$[11]$} \\
F18 & Lack of research on BIM implementation & {$[11]$} \\
F19 & Lack of BIM experts & {$[11]$} \\
F20 & Equipment purchase and maintenance costs & {$[12]$} \\
\hline & &
\end{tabular}

\section{Research methods}

Methods used in this study is the Decision-making Test and Evaluation Laboratory (DEMATEL). The DEMATEL method can calculate the influence degree and the affected degree of each element on other elements through the relationship between the elements in the system and the direct influence matrix. Then calculate the cause degree and the center degree of each element according to the previous step as the basis for constructing the model. So that it can determine the causal relationship between the elements and the position of each element in the system. According to this model, the main obstacles of BIM in prefabricated construction could be obtained and then draw influence. The steps of the research method are as follows:

Step 1: Through literature review, sort out and select 20 influencing factors that hinder the development of BIM in prefabricated construction, then make a questionnaire.

Step 2: This study uses the questionnaire survey method to quantify the relationships between the various obstacles, the former factor is considered to have an influence on the latter factor, and the " 1 " is considered to have no influence, and the " 0 " is considered to have no influence. The data was collected by distributing 60 questionnaires to personnel of construction units, managers, structural designers, scientific research institutes and other personnel engaged in the construction industry. A total of 44 valid questionnaires were collected in this survey, with an effective recovery rate of $73 \%$. 
Table 2. Division of direct influence between obstacles.

\begin{tabular}{lccccc}
\hline Proportion & 0 & $1-25$ & $26-50$ & $51-75$ & $76-100$ \\
\hline Score & 0 & 1 & 2 & 3 & 4 \\
\hline
\end{tabular}

Step 3: The questionnaire data are collected. Then the impact degree is divided according to the proportion of interviewees who think Fi has an impact on $\mathrm{Fj}$ (see Table 2).According to the scale of $0 \sim 4$, the direct influence degree between the obstacles is divided into 5 levels: none, small, normal, large and larger (the corresponding scores are 0, 1, 2, 3, 4 ). Through the data of the statistical questionnaire, the direct influence matrix $\mathrm{F}$ is obtained (see Table 3).

Step 4: Normalization is a routine operation to standardize things. So it needs to be calculated to the direct impact matrix normalization. The normalization matrix $\mathrm{X}$ is obtained by the following formula:

$$
X=\left(\frac{f_{i j}}{\operatorname{Max}}\right)_{n \times n} \quad(i \text { and } j \in 1,2,3, \Lambda, n) .
$$

Table 3. Direct influence matrix F.

\begin{tabular}{lllllllllllllllllllll}
\hline & F & F & F & F & F5 & F6 & F7 & F8 & F9 & F10 & F11 & F12 & F13 & F14 & F & F & F & F & F & F \\
& 1 & 2 & 3 & 4 & & & & & & & & & & & 1 & 1 & 1 & 1 & 1 & 2 \\
\hline F1 & 0 & 0 & 0 & 0 & 2 & 3 & 2 & 0 & 0 & 3 & 3 & 2 & 2 & 0 & 0 & 0 & 0 & 0 & 2 & 2 \\
F2 & 0 & 0 & 0 & 0 & 2 & 0 & 3 & 0 & 0 & 3 & 4 & 4 & 2 & 2 & 2 & 2 & 1 & 2 & 2 & 0 \\
F3 & 0 & 0 & 0 & 0 & 0 & 3 & 1 & 0 & 0 & 3 & 3 & 0 & 0 & 0 & 0 & 0 & 0 & 0 & 0 & 2 \\
F4 & 0 & 0 & 0 & 0 & 0 & 0 & 2 & 1 & 2 & 2 & 0 & 0 & 0 & 2 & 2 & 3 & 0 & 2 & 2 & 0 \\
F5 & 0 & 0 & 0 & 0 & 0 & 3 & 0 & 2 & 2 & 3 & 3 & 3 & 2 & 2 & 2 & 2 & 1 & 3 & 3 & 0 \\
F6 & 1 & 1 & 1 & 0 & 2 & 0 & 2 & 3 & 1 & 3 & 3 & 3 & 1 & 1 & 0 & 2 & 2 & 2 & 2 & 3 \\
F7 & 0 & 0 & 0 & 0 & 3 & 2 & 0 & 2 & 3 & 3 & 2 & 3 & 3 & 2 & 2 & 1 & 1 & 2 & 2 & 0 \\
F8 & 0 & 0 & 2 & 0 & 3 & 3 & 3 & 0 & 1 & 4 & 3 & 2 & 2 & 2 & 2 & 2 & 1 & 2 & 1 & 2 \\
F9 & 0 & 0 & 0 & 0 & 0 & 0 & 2 & 0 & 0 & 3 & 3 & 0 & 2 & 2 & 0 & 0 & 0 & 2 & 3 & 0 \\
F10 & 0 & 0 & 1 & 0 & 2 & 2 & 2 & 2 & 2 & 0 & 3 & 2 & 2 & 3 & 2 & 3 & 2 & 2 & 2 & 1 \\
F11 & 0 & 0 & 0 & 0 & 2 & 1 & 2 & 2 & 2 & 3 & 0 & 3 & 2 & 4 & 0 & 3 & 0 & 3 & 2 & 0 \\
F12 & 1 & 0 & 0 & 0 & 0 & 3 & 3 & 2 & 1 & 3 & 2 & 0 & 2 & 2 & 2 & 2 & 2 & 2 & 2 & 0 \\
F13 & 0 & 0 & 0 & 0 & 3 & 1 & 2 & 2 & 2 & 3 & 2 & 2 & 0 & 3 & 2 & 0 & 2 & 3 & 3 & 0 \\
F14 & 0 & 2 & 1 & 0 & 3 & 1 & 3 & 4 & 3 & 3 & 2 & 3 & 3 & 0 & 2 & 3 & 2 & 3 & 3 & 1 \\
F15 & 0 & 0 & 1 & 0 & 0 & 0 & 3 & 2 & 3 & 3 & 2 & 0 & 2 & 0 & 0 & 0 & 0 & 3 & 0 & 0 \\
F16 & 0 & 0 & 1 & 0 & 2 & 1 & 3 & 2 & 2 & 2 & 2 & 2 & 3 & 3 & 2 & 0 & 2 & 2 & 2 & 1 \\
F17 & 0 & 0 & 0 & 0 & 3 & 2 & 2 & 2 & 0 & 3 & 2 & 2 & 3 & 2 & 0 & 0 & 0 & 3 & 2 & 0 \\
F18 & 1 & 2 & 1 & 1 & 3 & 2 & 2 & 2 & 3 & 3 & 2 & 2 & 4 & 4 & 2 & 2 & 3 & 0 & 3 & 1 \\
F19 & 0 & 0 & 1 & 0 & 3 & 3 & 3 & 2 & 3 & 2 & 2 & 3 & 4 & 4 & 2 & 2 & 3 & 3 & 0 & 1 \\
F20 & 0 & 0 & 1 & 0 & 0 & 4 & 0 & 1 & 0 & 2 & 2 & 0 & 0 & 0 & 0 & 1 & 0 & 0 & 0 & 0 \\
\hline
\end{tabular}

Step 5: The next step is to calculate the total relation matrix $T$ :

$$
\mathrm{T}=\left(\mathrm{N}+\mathrm{N}^{2}+\mathrm{N}^{3}+\Lambda+\mathrm{N}^{\mathrm{K}}\right)=\sum_{\mathrm{k}=1}^{\infty} \mathrm{N}^{\mathrm{k}} \rightarrow \mathrm{T}=\mathrm{N}(\mathrm{I}-\mathrm{N})^{-1} .
$$


Step 6: According to the total relation matrix $T$, the influence degree and the affected degree of each element are further calculated. The influence degree is recorded as $D i$ and the affected degree is recorded as $\mathrm{Ci}$, and the following formula is obtained :

$$
D_{i}=\sum_{j=1}^{n} t_{i j},(i=1,2,3, \Lambda, n) ; \quad C_{i}=\sum_{j=1}^{n} t_{j i},(i=1,2,3, \Lambda, n) \text {. }
$$

Step 7: The center degree $M i$ and the cause degree $R i$ are calculated as following formulas:

$$
\mathrm{M}_{\mathrm{i}}=\mathrm{D}_{\mathrm{i}}+\mathrm{C}_{\mathrm{i}} ; \quad \mathrm{R}_{\mathrm{i}}=\mathrm{D}_{\mathrm{i}}-\mathrm{C}_{\mathrm{i}}
$$

\section{Research result}

\subsection{Center degree analysis}

According to the calculation of the data analysis software, the results are shown in Table 4. The data in Table 4 are formed in Fig.1. From the cause-result graph, the top five factors of center degree are F10, F18, F14, F19 and F11. Center degree indicates the position of the factor in the evaluation index system and its importance in the problem. Therefore, from the results of the center degree, it can be concluded that these five factors have greatly hindered the development of BIM technology in prefabricated construction. Among them, the conservative industry culture and resistance from client has the greatest impact, which reflects that the construction industry should currently be opened, and related customers should also support emerging technologies.

Table 4. Comprehensive influence relationship of obstacles.

\begin{tabular}{llllllllll}
\hline $\begin{array}{l}\text { Obstacl } \\
\text { es }\end{array}$ & $\mathrm{Di}$ & $\mathrm{Ci}$ & $\mathrm{Mi}$ & $\mathrm{Ri}$ & Obstacles & $\mathrm{Di}$ & $\mathrm{Ci}$ & $\mathrm{Mi}$ & $\mathrm{Ri}$ \\
\hline F1 & 0.8994 & 0.1425 & 1.0419 & 0.7570 & $\mathrm{~F} 11$ & 1.3238 & 1.8362 & 3.1600 & -0.5124 \\
F2 & 1.2870 & 0.2460 & 1.5330 & 1.0410 & $\mathrm{~F} 12$ & 1.2780 & 1.5731 & 2.8511 & -0.2952 \\
F3 & 0.4946 & 0.4502 & 0.9448 & 0.0444 & $\mathrm{~F} 13$ & 1.3408 & 1.7255 & 3.0663 & -0.3847 \\
F4 & 0.7988 & 0.0503 & 0.8491 & 0.7485 & $\mathrm{~F} 14$ & 1.7977 & 1.7231 & 3.5208 & 0.0747 \\
F5 & 1.3777 & 1.4758 & 2.8536 & -0.0981 & $\mathrm{~F} 15$ & 0.8139 & 1.0589 & 1.8728 & -0.2450 \\
F6 & 1.3942 & 1.4059 & 2.8001 & -0.0116 & $\mathrm{~F} 16$ & 1.3771 & 1.2308 & 2.6079 & 0.1462 \\
F7 & 1.3517 & 1.6649 & 3.0166 & -0.3132 & $\mathrm{~F} 17$ & 1.1971 & 1.0436 & 2.2407 & 0.1534 \\
F8 & 1.4735 & 1.4241 & 2.8976 & 0.0494 & $\mathrm{~F} 18$ & 1.8236 & 1.7153 & 3.5389 & 0.1083 \\
F9 & 0.7988 & 1.3817 & 2.1805 & -0.5828 & F19 & 1.7592 & 1.5607 & 3.3199 & 0.1986 \\
F10 & 1.4200 & 2.2008 & 3.6208 & -0.7809 & F20 & 0.4705 & 0.5682 & 1.0387 & -0.0976 \\
\hline
\end{tabular}

\subsection{Cause degree analysis}

If the cause degree is greater than 0 , it indicates that the factor has a greater influence than other factors, which is called the cause factor. Otherwise, it is called the result factor. The top five factors with positive cause degree are F2, F1, F4, F19 and F17, indicating that the lack of motivation and promotion has the greatest impact on other factors. The results also show that "Lack of intellectual property protection", "High precision requirements for fabricated components", "Lack of BIM experts", "Compatibility of BIM software" have medium influence on other factors. For example, a certain profit needs to be obtained in an assembly 
project for stakeholders. However, BIM technology and prefabricated construction are in the early stages of integration. The relevant national policies are not yet perfect, and stakeholders cannot see profits, which will make it more difficult for the market to adapt to the application of BIM technology.

The top five factors with negative cause degree are F10, F9, F11, F13 and F7. The results show that these factors will be greatly affected by other factors. From the above rankings, it can be concluded that the conservative industry culture and resistance from client is the most susceptible to other factors.

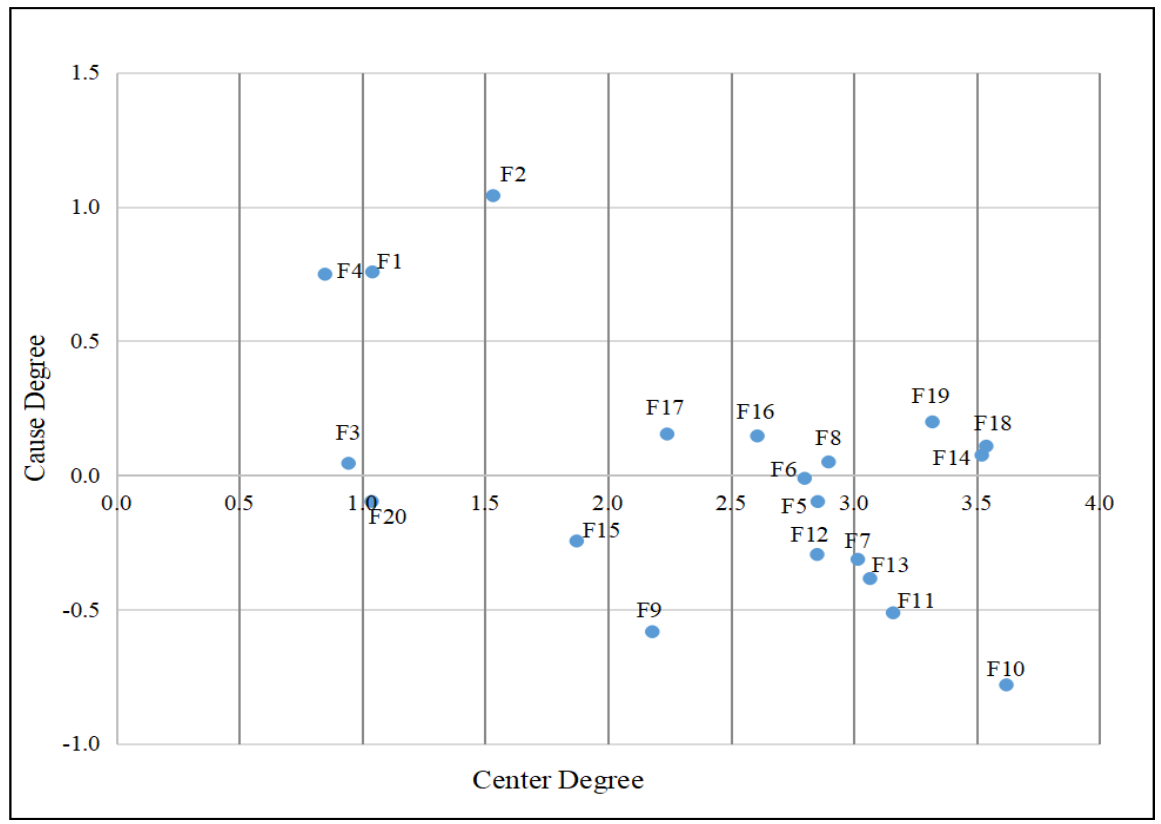

Fig.1. Cause-result graph.

\subsection{Discussion}

From the above calculation results, it can be seen that the conservative industry culture and resistance from client most likely to be affected by other factors, and this factor has the greatest obstacle to the development of BIM technology in prefabricated construction. And the biggest impact on other obstacles is the lack of corresponding incentives and promotion in China. Therefore, before solving the development of BIM technology in prefabricated construction, this factor should be solved first. To solve the problem of lack of corresponding incentives and promotion, the government should not simply formulate relevant incentive policies. But it needs to be practiced or simulated to calculate the cost of using BIM technology in prefabricated construction, and compare it with the cost of not using BIM technology. Find out where the increased costs of using BIM technology are then formulate targeted incentive and promotion policies for the increased costs. This will not only compensate for the loss of stakeholders using BIM technology in prefabricated construction, but also ensure that stakeholders can obtain a certain profit in it, so as to achieve the purpose of incentive promotion. Effective national policies will gradually open up the industry culture, and customers will gradually accept it. When the primary problem is solved, the secondary problem will be alleviated, so the impact of other obstacles will be reduced accordingly, and BIM technology will be more improved in prefabricated construction. 


\section{Conclusion}

Based on the previous research, this study uses the DEMATEL model to expand the obstacles to twenty. According to the calculation results of the model, first of all, the conservative industry culture and resistance from client has the greatest hindrance to the development of BIM technology in prefabricated construction, and is most susceptible to other factors. Secondly, the lack of research on BIM implementation, the lack of professional interaction, the lack of BIM experts, the negative treatment of stakeholders, and other factors also have hindered the development of BIM technology in prefabricated construction. Thirdly, the lack of incentives and promotion has the greatest impact on other factors. Therefore, the following suggestions can be adopted to appropriately reduce the obstacles to the development of BIM technology in China's prefabricated construction:

(1) Relevant policies formulated by the government for incentives and promotion can be more targeted to achieve the purpose of effectively making up for the losses of stakeholders, and allowing stakeholders to obtain certain profits by using BIM technology;

(2) The relevant laws and regulations should be improved to protect the intellectual property rights emerging technologies. The government should strengthen coordination and cooperation among departments, and hold more high-quality academic conference, in order to increase relevant study and interaction of BIM technology;

(3) The government can increase its encouragement and publicity to promote the cooperation between enterprises and universities to train BIM professionals in order to increase the enthusiasm of stakeholders.

Although this study is helpful for the further development of BIM technology in prefabricated construction, there are still two limitations. The first is that the DEMATEL method cannot rank the degree of obstruction of each factor, so it can only provide a general solution, and cannot provide detailed solution steps based on the problem. The second is that the scope of the questionnaire survey is only in Chengdu, the number of questionnaires in this study is insufficient. Therefore, future research directions should focus on major cities in China, expand the number of questionnaires and the scope of investigations, and consider adopting more comprehensive analytical methods to obtain more accurate conclusions.

\section{References}

1. X. Cao, X. Li, Y. Zhu, Z.J.J.o.c.p. Zhang, A comparative study of environmental performance between prefabricated and traditional residential buildings in China, J. Clean. Prod. 109 131-143 (2015).

2. T.U. Ganiron, Development and Efficiency of Prefabricated Building Components, Int. J. Smart Home, 10 85-94 (2016).

3. M. Oh, J. Lee, S.W. Hong, Y.J.A.i.c. Jeong, Integrated system for BIM-based collaborative design, AUTOMAT CONSTR, 58 196-206 (2015).

4. V. Jadhav, T. Nagwanshi, Y. Patil, D.J.v.-. Patil, IRJET, 2076-2078 (2017).

5. B. LIU, W.J.C.E. LIU, Application status and obstacles research of BIM in domestic construction industry, J. Constr. Econ., 9 (2015).

6. T.D. Oesterreich, F. Teuteberg, Behind the scenes: Understanding the socio-technical barriers to BIM adoption through the theoretical lens of information systems research, J.Technol. Forecast. Soc. Chang., 146 413-431 (2019).

7. J. Hong, G.Q. Shen, Z. Li, B. Zhang, W.J.J.o.c.p. Zhang, Barriers to promoting prefabricated construction in China: A cost-benefit analysis, J. Clean. Prod. ,172 649660 (2018). 
8. T. Tan, K. Chen, F. Xue, W.J.J.o.C.P. Lu, Barriers to Building Information Modeling (BIM) implementation in China's prefabricated construction: An interpretive structural modeling (ISM) approach, J. Clean. Prod. ,219 949-959 (2019).

9. F. Abanda, J. Tah, F.J.J.o.B.E. Cheung, BIM in off-site manufacturing for buildings, J. Build. Eng., 14 89-102 (2017).

10. [10] S. Azhar, M. Khalfan, T.J.C.E. Maqsood, Building information modelling (BIM): now and beyond, AJCEB, 12 15-28 (2012).

11. [11] J. Zhang, Y. Long, S. Lv, Y.J.P.e. Xiang, BIM-enabled modular and industrialized construction in China, Procedia Eng., 145 1456-1461 (2016).

12. [12] Y.-C. Lin, Y.-C.J.T.S.W.J. Su, Developing mobile-and BIM-based integrated visual facility maintenance management system, Sci. World J., 2013 (2013). 